\title{
Avaliação do estado nutricional correlacionado ao Aleitamento Materno em crianças de 5 a 10 anos no Município de Cascavel/PR
}

\author{
Evaluation of the nutritional status correlated to breastfeeding in children of 5 to 10 years in
} Cascavel/PR

Evaluación del estado nutricional correlacionado con la lactancia materna em niños de 5 a 10 años en la Ciudad de Cascavel/PR

Recebido: 25/03/2021 | Revisado: 30/03/2021 | Aceito: 29/04/2021 | Publicado: 13/05/2021

\author{
Laura Prado Godoy \\ ORCID: https://orcid.org/0000-0002-0857-3095 \\ Centro Universitário da Fundação Assis Gurgacz, Brasil \\ E-mail: laurapradogodoy@gmail.com \\ Anderson Dillmann Groto \\ ORCID: https://orcid.org/0000-0002-9028-4371 \\ Universidade Federal do Paraná, Brasil \\ E-mail: andersongr01@gmail.com \\ Marise Vilas Boas Pescador \\ ORCID: https://orcid.org/0000-0003-3718-1063 \\ Centro Universitário da Fundação Assis Gurgacz, Brasil \\ E-mail: marisevilasboas@hotmail.com
}

\begin{abstract}
Resumo
Introdução: O aleitamento materno apresenta inúmeros benefícios quando correlacionado ao desenvolvimento e crescimento infantil. A amamentação materna exclusiva até os seis meses proporciona inúmeros privilégios para o desenvolvimento do recém nato, benefícios estes que englobam desde o sistema imunológico até o estreitamento de laços familiares entre mãe e filho. Metodologia: Foram analisados, por meio de questionário estruturado, o estado nutricional atual e forma de nutrição durante a lactação de cento e cinquenta (150) crianças entre 5 a 10 anos. Resultados e Conclusão: Foi evidenciado que aleitamento materno exclusivo por pelo menos um mês é fator protetor para problemas relacionados ao peso (como baixo peso para a idade, sobrepeso ou obesidade) $(\mathrm{p}<0,001)$ e aleitamento por fórmula por pelo menos um mês é fator agravante para problemas relacionados ao peso $(\mathrm{p}<0,001)$.
\end{abstract}

Palavras-chave: Aleitamento materno; Estado nutricional; Obesidade infantil.

\begin{abstract}
Introduction: Breastfeeding has numerous benefits when correlated with child development and growth. Exclusive breastfeeding until six months provides numerous privileges that ranges from the immune system to family bonds between mother and child, benefiting the newborn's development. Methodology: 150 children aged 5 to 10 were analyzed using a structed questionnaire to assess their current nutritional status and form of nutrition during lactation. Results and Conclusion: Exclusive breastfeeding for at least one month is a protective factor for weight-related problems (such as underweight for age, overweight or obesity) $(\mathrm{p}<0,001)$ and formula breastfeeding for at least one month is an aggravating factor for weight-related problems $(\mathrm{p}<0,001)$.
\end{abstract}

Keywords: Breast Feeding; Nutritional status; Pediatric obesity.

\section{Resumen}

Introducción: La lactancia maternal tiene numerosos beneficios cuando se correlaciona con el desarrollo y crecimiento del niño. La lactancia maternal exclusive hasta los seis meses brinda numerosos privilegios para el desarrollo del recién nacido, beneficios que van desde el sistema inmunológico hasta el fortalecimiento de lazos familiares entre madre e hijo. Metodología: Se analizó el estado nutricional actual y la forma de nutrición durante la lactancia de ciento cincuenta (150) niños de 5 a 10 años mediante un cuestionario estructurado. Resultados y Conclusión: Se ha demostrado que la lactancia materna exclusiva durante al menos un mes es un factor protector para problemas relacionados con el peso (como bajo peso para edad, sobrepeso y obesidad) $(\mathrm{p}<0,001)$ y la lactancia materna con fórmula durante al menos un mes es factor agravante para problemas relacionados con el peso $(\mathrm{p}<0,001)$.

Palabras clave: Lactancia materna; Estado nutricional; Obesidad pediátrica. 


\section{Introdução}

A obesidade, uma doença crônica, de causa multifatorial, está intimamente atrelada as mais variadas condições mórbidas, sendo fator de risco para doenças cardiovasculares, respiratórias e metabólicas, apresentando uma difícil resolubilidade. Assim, o aumento da obesidade infantil é alarmante devido ao risco das crianças, futuramente, se tornarem adultos obesos (Balaban \& Silva, 2004).

Em decorrência da obesidade infantil diversas alterações metabólicas podem ser evidenciadas e associadas ao excesso de peso desde a primeira infância. Dentre as alterações correlacionadas ao excesso de peso tem-se as ortopédicas, as elevações dos triglicérides e colesterol, as dislipidemias, a hipertensão, diabetes, alterações no sistema cardiovascular, com alterações pressóricas, distúrbios respiratórios e até mesmo alterações dermatológicas que serão mais evidentes na vida adulta. Nota-se também distúrbios psicossociais e uma elevada taxa de mortalidade devido a doenças coronarianas naqueles indivíduos que foram obesos na infância e adolescência (Balaban \& Silva, 2004; Neves et al, 2010).

O aleitamento materno (AM) apresenta os mais variados benefícios para o desenvolvimento e crescimento infantil. Correlacionado ao desenvolvimento afetivo e intelectual, interfere de forma direta na redução da morbidade infantil, auxilia no desenvolvimento imunológico e atua como fator protetor para a obesidade infantil (Nunes, 2015; Ortega-García et al, 2018; Cordero et al, 2015; Yan et al, 2014).

Em virtude da dificuldade de tratar a obesidade em crianças e adolescentes e o risco que eventualmente estão associados a estas comorbidades torna-se imprescindível a identificação de estratégias eficazes para a prevenção, ressaltando as que acarretam menos efeito colateral. Assim, através do incentivo ao aleitamento materno é possível obter uma estratégia na prevenção da obesidade infantil, proteção essa reconhecida pela Organização Mundial de Saúde (OMS) (Siqueira \& Monteiro, 2007).

Levando-se em consideração o aumento da prevalência de obesidade na população e tendo como parâmetro o elevado índice de crianças e adolescentes com sobrepeso (SIMON et al, 2009), este estudo tem como objetivo analisar e correlacionar a associação do aleitamento materno ou alimentação via fórmula no período da lactação com o estado nutricional em crianças com idade entre cinco e dez anos.

\section{Obesidade infantil}

Obesidade pode ser compreendida como uma doença crônica de causa multifatorial em que ocorre um armazenamento excessivo de energia sob forma de triglicérides onde estes são armazenados no tecido adiposo. Sabe-se que através da ingestão e do gasto calórico é possível regular o estoque de energia do organismo e, consequentemente, o peso corporal (Rech et al, 2007; Wanderley \& Ferreira, 2010; Sigulem et al, 2001).

A obesidade é considerada um dos principais problemas de Saúde Pública nos países desenvolvidos e está cada vez mais tendo destaque nos países em desenvolvimento de forma coexistente com a desnutrição. Não sendo uma doença recente na literatura, a obesidade vem tomando proporções epidêmicas correlacionadas, principalmente, às mudanças no estilo de vida associada aos hábitos alimentares em que se observa aumento significativo no consumo de alimentos industrializados (Escrivão et al, 2000).

A biologia molecular está cada vez mais se destacando na elucidação quanto à fisiopatologia referente à obesidade através de estudos isolados de genes que controlam o balanço energético corporal podendo avaliar alterações no balanço energético acarretando ganho ou não de peso (Sigulem et al, 2001).

Sabe-se que a obesidade infantil é uma enfermidade crônica que poderá perdurar durante toda a vida do indivíduo, pois é estimado que $80 \%$ destas crianças permaneceram com sobrepeso na idade adulta, apresentando assim mais enfermidades metabólicas. Atrelada ao excesso calórico e hábitos de vida sedentários, a obesidade infantil está cada vez mais evidente. O leite 
materno apresenta fatores bioativos como hormônios e enzimas que irão atuar nos mais variados âmbitos do crescimento e maturação dos órgãos da criança. Estes fatores presentes no leite materno podem ser associados ao "imprinting metabólico" em que este é explicado por atuar de forma direta nos adipócitos alterando seu tamanho e quantidade. É necessário destacar a importância fundamental dos adipócitos em relação ao balanço enérgico, que quando alterado poderá ser um facilitador para a obesidade infantil. (ARAÚJO et al, 2006)

\section{Aleitamento materno como fator protetor}

A Organização Mundial de Saúde (OMS) recomenda como forma nutricional, até os seis meses de vida do lactante, o aleitamento materno de forma exclusiva devendo se estender de forma complementar até os dois anos de vida da criança. Diversos estudos apontam o efeito protetor advindo do leite materno contra a mortalidade infantil, variando de acordo com a idade da criança, a duração da amamentação e diversas características da população (OMS,1997).

As vantagens do aleitamento materno (AM) são inúmeras sendo tanto a curto quanto a longo prazo, visto que: "o leite materno é um alimento vivo, completo e natural, adequado para quase todos os recém-nascidos, excetuando raras exceções" (Levy \& Bértolo, 2008).

Sabe-se que aleitamento materno inclui muito mais variáveis que apenas aquelas relacionadas ao âmbito nutricional, englobando questões imunológicas e auxiliando no combate a diversas infecções, além de todas essas propriedades é possível evidenciar o estreitamento dos laços familiares entre mãe e filho. Vale ressaltar que o leite materno (LM) apresenta a quantidade nutricional ideal para o desenvolvimento do lactante, dentre estas propriedades deve-se destacar que a quantidade de lactose vigente no leite materno é a ideal para o desenvolvimento do sistema nervoso central, além disso o conteúdo hídrico do LM está em torno de $85 \%$ a $88 \%$ sendo a quantidade necessária para a hidratação da criança, mesmo nos dias mais quentes. Ainda referente a composição do leite materno, alguns autores destacam a baixa quantidade de sódio e níveis elevados de potássio como fatores protetores a longo prazo para desenvolvimento de doenças hipertensivas na vida adulta, citando ainda a presença de prostaglandinas provenientes do aleitamento materno que garantem uma maior motilidade do trato gastrointestinal, reduzindo a quantidade de vômitos, diarreias e refluxo. Outros estudos também apontam proteção contra o aparecimento do diabetes mellitus tipo I (Ferreira et al, 2011; Rea, 1990; Monte \& Giugliani, 2004).

Atrelando o aleitamento materno e a obesidade infantil é possível constatar que bebês que recebem amamentação materna exclusiva até os 6 meses e prolongam o período de lactação materna ganham menos peso comparados com aqueles que recebem outras fontes alimentícias durante o primeiro mês de vida. É possível constatar um aumento insulínico naquelas crianças que fazem uso de outros meios nutricionais podem desenvolver maior deposito de adipócitos, atuando de forma direta na obesidade infantil. Bebês alimentados de forma diferente do natural têm níveis circulantes de insulina mais elevados no sangue, como resultado do teor mais elevado de proteínas que estão presentes na fórmula infantil que, por sua vez, poderão estimular um depósito maior de gorduras (Amaral \& Basso, 2009).

Assim, é possível destacar que lactantes amamentados no seio materno são capazes de desenvolver mecanismos que serão capazes de controlar sua ingesta energética e até mesmo controlar sua alimentação através da mamadeira, possibilitando um mecanismo de autorregularão durante a mamada, evitando um sobrepeso ocasionado por uma ingesta calórica excessiva. (Simon et al, 2008)

\section{Metodologia}

Esta pesquisa de campo, prospectiva e de caráter quantitativo foi realizada através da aplicação de um questionário estruturado na Unidade Materno Infantil do Consórcio Intermunicipal do Oeste do PR - CISOP, utilizando como base metodológica o trabalho de Pereira et al (2018). 
Um questionário foi elaborado com quatro (4) questões objetivas e sete (7) dissertativas sendo aplicado apenas ao grupo alvo de estudo, o qual incluiu crianças entre 5 e 10 anos e suas mães que se propuseram a responder o questionário de forma voluntária assinando o Termo de Consentimento Livre e Esclarecido (TCLE) e sabiam informar a respeito da nutrição empregada durante o período de lactação.

O questionário tinha como objetivo levantar dados como data de nascimento para restringir a faixa etária desejada e o sexo para correlacioná-lo ao índice de sobrepeso.

Dados antropométricos foram obtidos através da balança Tanita Solar Scale 1632 para o peso e a estatura foi medida por meio do estadiômetro Seca Bodymeter 208.

Após a obtenção dos dados antropométricos o uso da calculadora, disponibilizada pela Rede Telessaúde Brasil, para o Índice de Massa Corpórea (IMC). Para o cálculo do IMC foi levado em consideração, além dos dados antropométricos, o sexo e a idade da criança em questão. Após realizado o cálculo em que se obtinha o IMC em Kg/m² e através de valores de referência foi possível classificar o estado nutricional da criança. Teve-se como parâmetro: Baixo IMC para idade: < percentil 3, IMC adequado ou eutrófico: $\geq$ Percentil 3 e $<$ Percentil 85, sobrepeso $\geq$ Percentil 85 e $<$ Percentil 97 e Obesidade $\geq$ Percentil 97.

A respeito do estado nutricional durante a lactação foram levantadas questões a respeito da amamentação materna exclusiva e durante quantas semanas essa prática foi empregada. Vinculado ao aleitamento materno foi questionado quando se iniciou a prática de introdução alimentar e por quantas semanas perdurou atrelada ao peito materno.

Levando em consideração sobre o estado nutricional também foi questionado se houve prática de lactação com fórmula de forma exclusiva e durante quantas semanas se manteve essa forma nutricional.

Tendo como base a demanda do serviço de saúde em questão teve-se como parâmetro para a realização da pesquisa a amostra de cento e cinquenta (150) participantes, os quais foram adequados aos critérios de inclusão e exclusão para o levantamento de dados.

\section{Resultados e Discussão}

Dados como sexo, altura, IMC, período de aleitamento materno exclusivo, período de aleitamento por fórmula e aleitamento materno complementado, obtidos a partir da aplicação do questionário, foram descritos na Tabela 1. 
Tabela 1: Informações referentes ao desenvolvimento de crianças entre 5 e 10 anos residentes um município do Oeste do Paraná $(\mathrm{n}=150)$

\begin{tabular}{|c|c|c|}
\hline Sexo & Total & \\
\hline Feminino & 72 & $48 \%$ \\
\hline Masculino & 78 & $52 \%$ \\
\hline \multicolumn{3}{|l|}{ Altura } \\
\hline Inferior ao percentil 5 & 3 & $2 \%$ \\
\hline Entre o percentil 5 e 95 & 113 & $75 \%$ \\
\hline Superior ao percentil 95 & 34 & $23 \%$ \\
\hline \multicolumn{3}{|l|}{ Classificação do IMC } \\
\hline Baixo IMC para a Idade & 2 & $1 \%$ \\
\hline Eutrófico & 78 & $52 \%$ \\
\hline Sobrepeso & 22 & $15 \%$ \\
\hline Obesidade & 48 & $32 \%$ \\
\hline \multicolumn{3}{|l|}{ Houve algum período de Aleitamento materno exclusivo? } \\
\hline Sim & 119 & $79 \%$ \\
\hline Não & 31 & $21 \%$ \\
\hline Mediana do tempo de aleitamento materno exclusivo & 20 & meses \\
\hline \multicolumn{3}{|l|}{ Houve algum período de Aleitamento exclusivo por fórmula? } \\
\hline Sim & 59 & $39 \%$ \\
\hline Não & 91 & $61 \%$ \\
\hline $\begin{array}{l}\text { Mediana do tempo de aleitamento exclusivo por } \\
\text { fórmula }\end{array}$ & 20 & meses \\
\hline \multicolumn{3}{|l|}{ Houve algum período de Aleitamento materno complementado? } \\
\hline Sim & 65 & $43 \%$ \\
\hline Não & 85 & $57 \%$ \\
\hline $\begin{array}{l}\text { Mediana do tempo de aleitamento materno } \\
\text { complementado }\end{array}$ & 58 & meses \\
\hline
\end{tabular}

Fonte: Autores (2021).

As correlações que obtiveram significância estatística foram descritas na Tabela 2. Houve correlação entre aleitamento materno exclusivo por pelo menos um mês com proteção em problemas relacionados ao peso (p<0,001), Razão de Chances $(\mathrm{OR})$ de $0.24(0.10-0.59)$ e aleitamento por fórmula por pelo menos um mês com maior prevalência de problemas relacionados ao peso $(\mathrm{p}<0.001)$, com OR de $3.88(1.94-7.78)$. 
Tabela 2: Correlações estatisticamente significativas $(\mathrm{p}<0,05)$

Hipótese Alternativa
Valor $\mathbf{p}$

Odds Ratio (OR) e Intervalo de confiança $(95 \%)$

Aleitamento materno exclusivo por pelo menos um mês é um fator protetor para problemas relacionados ao peso (baixo peso para a idade, sobrepeso ou obesidade) na comparação com crianças que não tiveram aleitamento materno

Aleitamento materno exclusivo por pelo menos um mês é um fator protetor para problemas relacionados ao peso (baixo peso para a idade, sobrepeso ou obesidade) em meninos, na comparação com meninos que não tiveram aleitamento materno

Aleitamento por fórmula por pelo menos um mês é um fator agravante para problemas relacionados ao peso (baixo peso para a idade, sobrepeso ou obesidade) na comparação com crianças que não tiveram aleitamento por fórmula

Aleitamento por fórmula por pelo menos um mês é um fator agravante para problemas relacionados ao peso (baixo peso para a idade, sobrepeso ou obesidade) em meninos, na comparação com meninos que não tiveram aleitamento por fórmula

Aleitamento por fórmula por pelo menos um mês é um fator agravante para problemas relacionados ao peso (baixo peso para a idade, sobrepeso ou obesidade) em meninas, na comparação com meninas que não tiveram aleitamento por fórmula
$<0.001 \quad 0.24(0.10-0.59)$

$<0.001$

$0.21(0.07-0.66)$

$<0.001$

$3.88(1.94-7.78)$

0.01

$3.75(1.44-9.78)$

$4.00(1.45-11.01)$

Fonte: Pesquisadores (2021).

\section{Considerações Finais}

A obesidade infantil, por ser considerada um problema de saúde pública e levando-se em consideração o fato de ser caracterizada como uma doença crônica que predispõem inúmeros malefícios a curto e longo prazo, deve ser estudada para consolidar fatores protetores e de risco associados estatisticamente a esta comorbidade.

Este trabalho tem como objetivo descrever benefícios provenientes da alimentação materna e dentre eles salientar o fator protetor contra obesidade infantil e outros problemas relacionados ao peso. Foi observado através dos dados levantados que crianças que tiveram aleitamento materno como forma nutricional exclusiva apresentam mais chances de terem ganho ponderal adequado ( $p<0,001)$ na comparação com crianças alimentadas por meio de fórmula (Tabela 2).

Em contrapartida, quando correlacionado o aleitamento através de fórmula como fator nutricional aos lactantes foi possível evidenciar esta forma de nutrição como um fator agravante para problemas relacionados ao peso $(\mathrm{p}<0,001)$ em todas as crianças e também quando considerando apenas os subgrupos de meninos ( $\mathrm{p}=0,01)$ e meninas $(\mathrm{p}=0,01)$.

Futuros trabalhos podem abordar o acompanhamento de crianças obesas que não receberam aleitamento materno, avaliando se estas terão maior prevalência de doenças isquêmicas cerebrovasculares, depressão e doenças de Parkinson e Alzheimer na comparação com crianças obesas que receberam aleitamento materno.

Portanto, para combater a obesidade infantil e as comorbidades futuras decorrentes desta patologia, faz-se necessário implementar estratégias e planos para a promoção e apoio a amamentação materna de forma exclusiva perdurando nos primeiros seis meses de vida e complementado até os dois anos da criança, conforme indicado pela Organização Mundial de Saúde (OMS, 1997). 


\section{Referências}

Amaral, S. do, Basso, C. (2009). Aleitamento Materno e Estado Nutricional Infantil. Disciplinarum Scientia, 10(1), 19-30.

Araújo, M. F. M. de, Beserra, E. P., \& Chaves, E. S. (2006). O papel da amamentação ineficaz na gênese da obesidade infantil: um aspecto para a investigação de enfermagem. ACTA Paulista de Enfermagem, 19(4), 450-455.

Balaban, G., \& Silva, G. A. P. (2004). Efeito protetor do aleitamento materno contra a obesidade infantil. Jornal de Pediatria, 80(1), 7-16.

Bastos, C. de O., Salim, T. R., Do Carmo, A. L. O., Muratori, A. S., Nogueira, É. de T., Munhoz, E. K. et al (2020). Deficiência do aleitamento materno exclusivo como contribuinte para a obesidade infantil. Revista Eletrônica Acervo Científico, 17, e5757.

Cordero, M. J. A., Sánchez Lòpez, A. M., Baños, N. M., Villar, N. M., Ruiz, M. E., \& Rodrìguez, E. H. (2015). Lactancia materna como prevención del sobrepeso y la obesidad en el niño y el adolescente; revisión sistemática. Nutricion Hospitalaria, 31(2), 606-620. https://doi.org/10.3305/nh.2015.31.2.8458

Escrivão, M. A. M., Oliveira, F. L., Tadder, J. A. de A., \& Ancona Lopez, F. (2000). Obesidade exógena na infância e na adolescência. Jornal de Pediatria, $5(2), 39-42$.

Ferreira, M., Nelas, P., \& Duarte, J. (2011). Motivação para o aleitamento materno: Variáveis intervenientes. Millenium, 40, $23-38$.

Levy, L., \& Bértolo, H. (2008). Manual de Aleitamento Materno.

Monte, C. M. G., \& Giugliani, E. R. J. (2004). Recomendações para alimentação complementar da criança em aleitamento materno. Jornal de Pediatria, 80(5), $131-141$.

Neves, P. M., Torcato, A., Urquieta, A., \& Kleiner, A. F. (2010). Importância do tratamento e prevenção da obesidade infantil. Arq. Ciênc. Saúde, 4, 150-153.

Nunes, L. M. (2015). Importância do aleitamento materno na atualidade. Boletim Científico de Pediatria, 4(3), 55-58.

Organização Mundial da Saúde. (1997). Normas Alimentares para Crianças Brasileiras Menores de dois anos: Embasamento Científico.

Ortega-Garciá, J. A., Kloosterman, N., Alvarez, L., Tobarra-Sánchez, E., Cárceles-Álvarez, A., Pastor-Valero, R., López-Hernández, F. A., Sánchez-Solis, M., \& Claudio, L. (2018). Full Breastfeeding and Obesity in Children: A Prospective Study from Birth to 6 Years. Childhood Obesity, 14(5), 327-337. https://doi.org/10.1089/chi.2017.0335

Pereira A. S. et al. (2018). Metodologia da pesquisa científica. [free e-book]. Santa Maria. Ed. UAB/NTE/UFSM

Rea, M. F. (1990). The Brazilian national breastfeeding program: A success story. International Journal of Gynecology and Obstetrics, 31(SUPPL. 1), 79-82.

Rech, R. R., Halpern, R., Mattos, A. P. de, Bergmann, M. L. de A., Costanzi, C. B., \& Alli, L. R. (2007). Obesidade infantil: complicações e fatores associados. Sigulem, D. M., \& Devincenzi, M. U. (2008). Obesidade na Infância e na Adolescência.

Simon, V. G. N., Souza, J. M. P. de, \& Souza, S. B. de. (2008). Breastfeeding, complementary feeding, overweight and obesity in pre-school children. Revista de Saúde Pública, 43(1), 1-9.

Siqueira, R. S. de, \& Monteiro, C. A. (2007). Amamentação na infância e obesidade na idade escolar em famílias de alto nível socioeconomico. Revista de Saude Publica, 41(1), 5-12.

Wanderley, E. N., \& Ferreira, V. A. (2010). Obesidade: uma perspectiva plural Obesity: a plural perspective. Ciência \& Saúde Coletiva, 15(1), $185-194$.

Yan, J., Liu, L., Zhu, Y., Huang, G., \& Wang, P. P. (2016). The association between breastfeeding and childhood obesity: A meta-analysis. World Review of Nutrition and Dietetics, 114, 110-111. https://doi.org/10.1159/000441820 